\title{
Analysis of the Performance of Diesel Engine Fueled using B50-B100 Biodiesel Based on Simulation
}

\author{
Semin $^{1}$, Beny Cahyono 2 , Himmawan Aan Listyanto ${ }^{3}$, Rosli Abu Bakar ${ }^{4}$ \\ (Received: 19 August 2020 / Revised: 20 September 2020 / Accepted: 22 September 2020)
}

\begin{abstract}
In addition, the purpose of this study is to determine the effect of waste cooking oil biodiesel blends in the performance testing of a onecylinder diesel engine simulation modeling. The method used by the author in this study is to use a simulation method. Performance-based diesel motor performance tests are performed using HSD, and also with variations of used cooking oil biodiesel fuel mixtures. From the performance test results at full load, it was found that the comparison of the value of the power mix of biodiesel waste cooking oil with HSD decreased power. At B50 decreased power (6.38\%), B60 (7.6\%), B70 $(8.9 \%)$, B80 (10.2\%), B90 (11.4\%), and B100 (12.7\%) at maximum RPM. The torque value obtained in the biodiesel fuel mixture also decreased compared to HSD in the same cycle. The lowest SFOC value is produced by HSD fuel. SFOC HSD value is lower than cooking oil biodiesel mixture which is higher $(6.8 \%)$ B50 fuel, up (8.3\%) B60, up (9.83\%) B70, up $(11.4 \%)$ B80, up (12.9\%) B90, and up (14.5\%) B100 at full load and maximum RPM conditions.
\end{abstract}

Keywords — biodiesel, performance test, waste cooking oil.

\section{INTRODUCTION}

Oil fuels have an important role in the development of the transport sector, industry growth, and agriculture sector and to meet other human needs. But the total of oil fuel orders in the world, causing shortening on the day reserves of fossil fuels available. Because of that, most scientists and researchers are looking for a substitute fuel or alternative [1]. The lack of fossil fuel deposits will make renewable fuel energy more attractive [2]. Whereas most renewable energy that has energy technology is more environmentally friendly than conventional energy options, because of its very slow acceptance factors such as lack of supply, economic constraints, etc.

President of the Republic of Indonesia Joko Widodo [3], in his statement, the president said that if in this B30 should reduce the import of diesel fuel seriously. Governments are expected to reduce the number of Diesel fuel imports because this B30 program can make a very large donation because it can save the country's foreign exchange to Rp63 trillion-Indonesia's largest oil palm producer in the world. We have a source of vegetable material as a substitute for Diesel fuel. We must use this for social energy independence. In this B30 program will create a huge domestic CPO demand. This

Semin, Departement of Marine Engineering, Institut Teknologi Sepuluh Nopember, Surabaya, 60111, Indonesia.

E-mail: seminits@yahoo.com

Beny Cahyono, Department of Marine Engineering, Institut Teknologi Sepuluh Nopember, Surabaya, 60111, Indonesia.

E-mail: benyjtsp88@gmail.com

Himmawan Aan Listyanto, Departement of Marine Engineering, Institut Teknologi Sepuluh Nopember, Surabaya, 60111, Indonesia.

E-mail: himmawanska@gmail.com

Rosli Abu Bakar, Faculty of Mechanical Engineering, University Malaysia Pahang, Pekan - Pahang, Malaysia.

E-mail: rosli@ump.edu.my means B30 will have a direct impact on the small plantations that foster people farmers who have worked in the palm oil plantation. The B30 Program will later become B50, and so on. It could even be B100.

Biodiesel is an ingredient that can be used as a substitute for diesel engine fuel. This is due to raw materials derived from vegetable oil that can be renewed, can be produced periodically, and easily obtained. In addition, the price is relatively stable, and the production is easy to adjust to needs. Biodiesel is also an environmentally friendly fuel. It does not contain sulfur so as to reduce the environmental damage caused by acid rain [4].

The development of renewable energy has been much, which is done research on the diesel engine fuel testing that has been done. For example, biodiesel is derived from Jatropha castor oil [5]. In oil, palm oil has also been tested on research [6]. The government has also commercialized biodiesel through Pertamina, but using oil palm biodiesel mixture. The commercialized biodiesel is a mixture of diesel with biodiesel, commonly known as bio diesel fuel.

Waste cooking oil is a biodiesel material in addition to the potential of palm oil to become a biodiesel fuel. The advantage of its use, in addition to its low price, can also cope with pollution caused by waste cooking oil waste [7].

This research tests the performance of Waste cooking oil biodiesel blends. However, the characteristics of used cooking oil biodiesel properties, it has been researched previously with reference to Indonesian National Standard 2015 (SNI), only limited its characteristic test. From there, the authors conducted further research on the performance test of used cooking oil that was simulated into the GT-POWER application. Thus, future performance tests will support the use of biodiesel B50-B100 as alternative fuels when in commercial production. 


\section{METHOD}

The method used in this research is to use a simulated method on a diesel engine. The modeling of diesel engines used in this simulation uses Yanmar engine $85 \mathrm{MH}$. The variety of fuel used is the use of used cooking oil biodiesel B50, B60, B70, B80, B90, B100. Before the simulation begins, calibration will be done by comparing the simulation result with engine specifications Yanmar TF $85 \mathrm{MH}$ to know the performance of the diesel engine. . Thus, it can be assumed that the performance of the machine at the time of calibration is initial performance.

\section{A. Engine Diesel Simulation Modeling}

Diesel engine simulation modeling is done by measuring the diesel engine that has been designed. Diesel engine modeling Data using engine specifications Yanmar TF $85 \mathrm{MH}$. Table 1 shows the machine specification data.

TABLE 1.

ENGINE DATA SPECIFICATION YANMAR TF $85 \mathrm{MH}$

\begin{tabular}{ll}
\hline Engine Data & Specification \\
\hline Engine Type & Diesel engine \\
Power Maximum & $8,5 \mathrm{HP} 2200 \mathrm{RPM}$ \\
Torque Maximum & $3,44 \mathrm{~kg} . \mathrm{m} / 1600$ \\
& RPM \\
SFOC & $171 \mathrm{gr} / \mathrm{hp}$. hours \\
Power Continue & $7,5 \mathrm{HP} / 2200 \mathrm{RPM}$ \\
Type of Stroke & 4 Stroke \\
System of Combustion & Direct injection \\
Compression Ratio & 18 \\
Injection Pressure & $200 \mathrm{~kg} / \mathrm{cm}^{3}$ \\
Cooling System & Hopper \\
Oil Type & SAE 40 Class CC \\
& or CD \\
Dimension of Engine (Mm) & 672 X 330,5 X 496 \\
Number of Cylinder & $1 \mathrm{cylinder}$ \\
Diameter X Length & $85 \mathrm{~mm} \mathrm{X} 87 \mathrm{~mm}$ \\
Start of Injection & $18^{\circ}$ before TMA \\
Cylinder Volume & $493 \mathrm{cc}$ \\
\hline
\end{tabular}

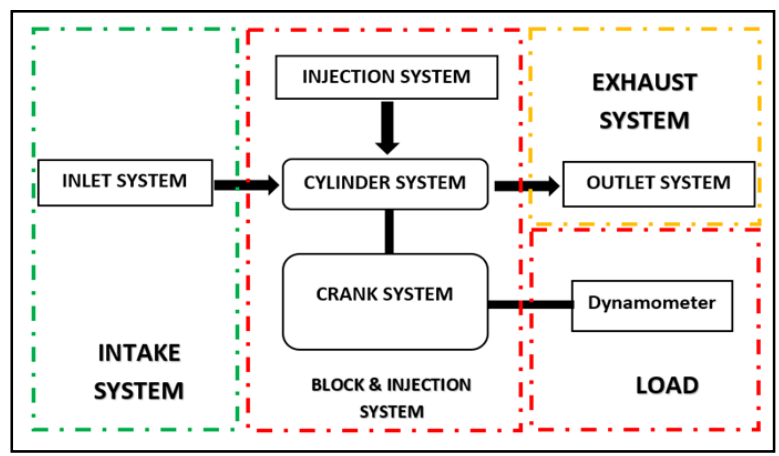

Figure. 1. Schematic of Diesel Engine

Engine specification Data Yanmar TF 85 used for modeling design in diesel engine simulation as per table 1 IE engine specification data Yanmar TF 85. There are several stages in modeling creation as follows:

\section{1) Measurement of Engine Dimension}

The engine modeling stage, diesel engine dimension data, has been measured in accordance with the engine specification Yanmar TF 85.

\section{2) Engine Modeling Schematic}

Figure 1 shows the diesel engine scheme of Yanmar TF $85 \mathrm{MH}$. This scheme is divided into three main sections. The exhaust system, both the block system and the injector, the inlet system.

\section{3) Component Definition Simulation}

The manufacture of the diesel engine simulation modeling is required data Engine specification parameters from Project Guide machines. There are several parts of the object that must be defined so that the simulation generates output data in the form of machine performance as well as possible with comparative specification data. There are three main systems, namely the exhaust gas system, cylinder block system, and injector, inlet system.

A. Components in the exhaust system are several stages that must be defined according to the specifications of Yanmar diesel engine 85, as follows: in the exhaust system, there are several steps that must be defined according to the Yanmar TF 85 diesel engine specifications, as follows:

\section{Exhaust Valve}

This component has the same function as the inlet valve. The Exhaust Valve is made by inputting the diameter of the valve, valve lash, cam timing angle, and elevator arrays.

\begin{tabular}{|c|c|c|c|c|}
\hline Timing & \multirow{2}{*}{$\begin{array}{l}\sim \text { Flow Coefficients } \\
\text { Attribute }\end{array}$} & \multicolumn{3}{|l|}{$\checkmark$ Output 圆 Plots } \\
\hline & & Unit & & \\
\hline \multicolumn{2}{|c|}{ Cam Timing Angle } & Cam Angle $\vee$ & & $141 \ldots$ \\
\hline \multicolumn{2}{|c|}{ Cam Timing Anchor Reference } & & TDCFiring & $\checkmark$ \\
\hline \multicolumn{2}{|r|}{ Cam Timing Lift Array Reference } & & Theta $=0$ & $\checkmark$ \\
\hline \multicolumn{5}{|c|}{ Source of Angle } \\
\hline (-) & \multicolumn{4}{|l|}{ Attached Cylinder } \\
\hline 0 & Part on Map & & & ign \\
\hline 0 & \multicolumn{2}{|l|}{ Driver Reference Object } & & ign \\
\hline
\end{tabular}

Figure. 2. Part of Timing Exhaust Valve

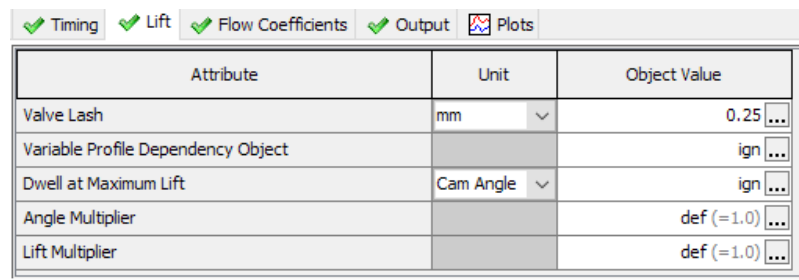

Figure. 3. Part of Lift Exhaust Valve 


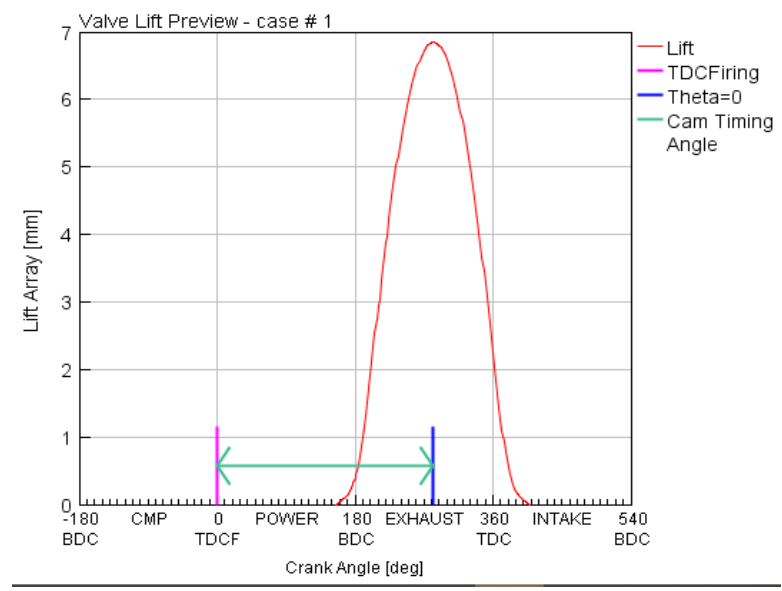

Figure. 4. Part of Lift Exhaust Valve

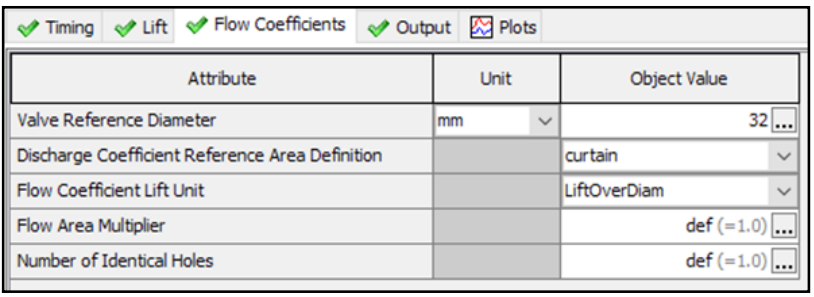

Figure. 5. Part of Flow Coefficient Exhaust Valve

\section{Exhaust Port System}

It is a pipe (cylinder) that serves to connect the exhaust valve with an exhaust runner. Data filled in diameter, length, material, hardness value, and temperature

\begin{tabular}{|c|c|c|c|c|c|c|c|}
\hline \multicolumn{2}{|c|}{ Main } & Thermal & \multicolumn{5}{|c|}{ Pressure Drop 图 Plots } \\
\hline & \multicolumn{3}{|r|}{ Attribute } & \multicolumn{2}{|c|}{ Unit } & \multicolumn{2}{|c|}{ Object Value } \\
\hline \multicolumn{8}{|c|}{ Basic Geometry and Initial Conditions } \\
\hline \multicolumn{4}{|c|}{ Diameter at Inlet End } & $\mathrm{mm}$ & 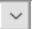 & & $38.5873 \ldots$ \\
\hline \multicolumn{4}{|c|}{ Diameter at Outlet End } & $\mathrm{mm}$ & $v$ & & $30.78 \ldots$ \\
\hline \multicolumn{4}{|c|}{ Length } & $\mathrm{mm}$ & 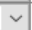 & & $30.2 \ldots$ \\
\hline \multicolumn{4}{|c|}{ Discretization Length } & $\mathrm{mm}$ & 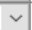 & & $46.75 \ldots$ \\
\hline \multicolumn{4}{|c|}{ Initial State Name } & & & & Gas_buang .... \\
\hline \multicolumn{8}{|c|}{ Surface Finish } \\
\hline 0 & \multicolumn{3}{|c|}{ Smooth } & & & & \\
\hline (-) & \multicolumn{3}{|c|}{ Roughness from Material } & & & cast_iron & $\checkmark$ \\
\hline 0 & \multicolumn{3}{|c|}{ Sand Roughness } & $\mathrm{mm}$ & $\checkmark$ & & \\
\hline \multicolumn{8}{|c|}{ Additional Geometry Options } \\
\hline \multicolumn{4}{|c|}{ Radius of Bend } & mm & $\sim$ & & $\operatorname{ign} \ldots$ \\
\hline \multicolumn{4}{|c|}{ Angle of Bend } & deg & $\checkmark$ & & ign $\ldots$ \\
\hline \multicolumn{4}{|c|}{ Pipe Elevation Change or 3D Acceleration Object } & $\mathrm{mm}$ & $\checkmark$ & & $\operatorname{ign}[. .$. \\
\hline \multicolumn{4}{|c|}{ Number of Identical Pipes } & & & & $\operatorname{def}(=1,0) \ldots$ \\
\hline
\end{tabular}

Figure. 6. Main Part of Exhaust Port

\begin{tabular}{|c|c|c|c|}
\hline \multicolumn{4}{|c|}{ Main Thermal $\checkmark$ Pressure Drop $⿴$ Plots } \\
\hline & Attribute & Unit & Object Value \\
\hline \multicolumn{4}{|c|}{ Wall Temperature Method } \\
\hline (-) & Imposed Wall Temperature & $\checkmark$ & $489 \ldots$ \\
\hline O & Calculated Wall Temperature & & \\
\hline O & Wall Temperature from Connected Thermal Primitive & & \\
\hline $\mathrm{O}$ & Adiabatic & & \\
\hline \multicolumn{4}{|c|}{ Additional Thermal Options } \\
\hline \multicolumn{2}{|c|}{ Heat Transfer Multiplier } & & $1.5 \ldots$ \\
\hline \multicolumn{2}{|c|}{ Heat Input Rate } & w & $\operatorname{ign} \ldots$ \\
\hline \multicolumn{2}{|c|}{ Thermocouple Object } & & $\operatorname{ign} \ldots$ \\
\hline (-) & \multicolumn{3}{|l|}{ Heat Transfer Correlation (Colburn) } \\
\hline O & User Defined Heat Transfer Model & & ign \\
\hline $\mathrm{O}$ & Heat Transfer Coefficient & $W /\left(m^{\wedge} 2-K\right) \vee$ & \\
\hline \multicolumn{2}{|r|}{ Condense/Evaporate Water Vapor (Non-Refrigerant Circuits) } & & off \\
\hline
\end{tabular}

Figure. 7. Thermal Part of Exhaust Port

\begin{tabular}{|c|c|c|c|c|c|c|}
\hline \multirow[t]{2}{*}{$\diamond M$} & \multicolumn{6}{|c|}{ lain $\leadsto$ Thermal $\leadsto$ Pressure Drop $⿴ 囗$ Plots } \\
\hline & \multicolumn{2}{|r|}{ Attribute } & \multicolumn{2}{|c|}{ Unit } & \multicolumn{2}{|r|}{ Object Value } \\
\hline \multicolumn{7}{|c|}{ Basic Geometry and Initial Conditions } \\
\hline \multicolumn{3}{|c|}{ Diameter at Inlet End } & $\mathrm{mm}$ & $\checkmark$ & & $38.44 \ldots$ \\
\hline \multicolumn{3}{|c|}{ Diameter at Outlet End } & $\mathrm{mm}$ & $\checkmark$ & & $38.1 \ldots$ \\
\hline \multicolumn{3}{|c|}{ Length } & $\mathrm{mm}$ & $\checkmark$ & & $152.7 \ldots$ \\
\hline \multicolumn{3}{|c|}{ Discretization Length } & $\mathrm{mm}$ & $v$ & & $32.4 \ldots$ \\
\hline \multicolumn{3}{|c|}{ Initial State Name } & & & & Gas_buang .... \\
\hline \multicolumn{7}{|c|}{ Surface Finish } \\
\hline O & \multicolumn{6}{|c|}{ Smooth } \\
\hline (0) & \multicolumn{2}{|c|}{ Roughness from Material } & & & \multicolumn{2}{|l|}{ steel } \\
\hline O & Sand $\mathrm{R}$ & Roughness & $\mathrm{mm}$ & $\checkmark$ & & \\
\hline \multicolumn{7}{|c|}{ Additional Geometry Options } \\
\hline \multicolumn{3}{|c|}{ Radius of Bend } & $\mathrm{mm}$ & $\checkmark$ & & $\operatorname{ign} \ldots$ \\
\hline \multicolumn{3}{|c|}{ Angle of Bend } & deg & $\checkmark$ & & $\operatorname{ign} \ldots$ \\
\hline \multicolumn{3}{|c|}{ Pipe Elevation Change or 3D Acceleration Object } & $\mathrm{mm}$ & $\checkmark$ & & $\operatorname{ign}[.$. \\
\hline \multicolumn{3}{|c|}{ Number of Identical Pipes } & & & & $\operatorname{def}(=1,0) \ldots$ \\
\hline
\end{tabular}

Figure. 8. Main Part of Exhaust Runner

\begin{tabular}{|c|c|c|c|}
\hline \multicolumn{4}{|c|}{$\triangle$ Main $\leadsto$ Thermal $\&$ Pressure Drop 图 Plots } \\
\hline & Attribute & Unit & Object Value \\
\hline \multicolumn{4}{|c|}{ Wall Temperature Method } \\
\hline c & Imposed Wall Temperature & k & $4 8 9 \longdiv { . . . }$ \\
\hline C & Calculated Wall Temperature & & \\
\hline C & Wall Temperature from Connected Thermal Primitive & & \\
\hline C & Adiabatic & & \\
\hline \multicolumn{4}{|c|}{ Additional Thermal Options } \\
\hline \multicolumn{2}{|c|}{ Heat Transfer Multiplier } & & $\operatorname{def}(=1,0) \ldots$ \\
\hline \multicolumn{2}{|c|}{ Heat Input Rate } & & $\operatorname{ign}[. .$. \\
\hline \multicolumn{2}{|c|}{ Thermocouple Object } & & $\operatorname{ign} \ldots$ \\
\hline & Heat Transfer Correlation (Colburn) & & \\
\hline & User Defined Heat Transfer Model & & ign \\
\hline & Heat Transfer Coeffficent & $w /\left(m^{\wedge} 2+k\right) \sim$ & \\
\hline & dense/Evaporate Water Vapor (NonRRefigerant Circuits) & & 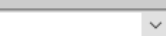 \\
\hline
\end{tabular}

Figure. 9. Main Part of Thermal Exhaust Runner

\section{End Environment}

The last component with the environment boundary condition of the exhaust gas combustion system. The Data is inserted, such as an inlet environment that determines the pressure value, temperature, and air composition of combustion results.

\begin{tabular}{|l|l|r|r|}
\hline \multicolumn{1}{|c|}{ Attribute } & \multicolumn{2}{|c|}{ Unit } & \multicolumn{1}{|c|}{ Object Value } \\
\hline Pressure (Absolute) & bar & $\checkmark$ & $1 \ldots$ \\
\hline Temperature & $\mathrm{K}$ & $\checkmark$ & $303.15 \ldots$ \\
\hline Composition & & & air $\ldots$ \\
\hline
\end{tabular}

Figure. 10. Main Part of End-outlet

B. Some components must be specified in the cylinder, crank train, and injection system. As follows:

\section{Engine Cylinder}

Inside the engine cylinder, some input data should be included, namely the cylinder wall temperature, fluid flow, heat transfer, combustion model. 


\begin{tabular}{|c|c|c|c|}
\hline \multicolumn{4}{|c|}{$\checkmark$ Main $\checkmark$ Advanced $ه$ Plots } \\
\hline & Attribute & Unit & Object Value \\
\hline \multicolumn{2}{|c|}{ Initial State Object } & & initial $[. .$. \\
\hline () & Wall Temperature defined by Reference Object & & twall $[\ldots$ \\
\hline 0 & Wall Temperature defined by FE Structure part (EngCyISt... & & \\
\hline \multicolumn{2}{|c|}{ Heat Transfer Object } & & ht $[. .$. \\
\hline \multicolumn{2}{|c|}{ Flow Object } & & Piston_Yanmar__Standart ... \\
\hline \multicolumn{2}{|c|}{ Combustion Object } & & comb $\ldots$ \\
\hline \multicolumn{2}{|r|}{ Measured Cylinder Pressure Analysis Object } & & $\operatorname{ign}[. .$. \\
\hline \multicolumn{2}{|r|}{ Cylinder Pressure Analysis Mode } & & off \\
\hline
\end{tabular}

Figure. 11. Main Part of Cylinder

\begin{tabular}{|c|c|c|c|}
\hline$\checkmark$ Main $\checkmark$ Altitude and Humidity & & & \\
\hline Attribute & & & Object Value \\
\hline Pressure (Absolute) & bar & $v$ & $1 \ldots$ \\
\hline Temperature & c & $v$ & $30 \ldots$ \\
\hline Composition & & & air $\ldots$ \\
\hline
\end{tabular}

Figure. 12. Main Part of Initial State Object

\begin{tabular}{|c|c|c|c|}
\hline \multicolumn{4}{|l|}{$\checkmark$ Main } \\
\hline Attribute & \multicolumn{2}{|c|}{ Unit } & Object Value \\
\hline Head Temperature & K & $\vee$ & $523.15 \ldots$ \\
\hline Piston Temperature & K & $\checkmark$ & $523.15 \ldots$ \\
\hline Cylinder Temperature & K & $\checkmark$ & $403.15 \ldots$ \\
\hline
\end{tabular}

Figure. 13. Main Part of Wall Temperature

\begin{tabular}{||l|l|r|r||}
\hline \multicolumn{1}{|c|}{ Attribute } & \multicolumn{2}{c|}{ Unit } & \multicolumn{2}{|c|}{ Object Value } \\
\hline Piston Cup Diameter (Maximum) & $\mathrm{mm}$ & $\checkmark$ & \\
\hline Piston Cup Depth at Maximum Diameter & $\mathrm{mm}$ & $\checkmark$ & \\
\hline Piston Cup Diameter (Edge) & $\mathrm{mm}$ & $\checkmark$ & \\
\hline Piston Cup Center Depth & $\mathrm{mm}$ & $\checkmark$ & \\
\hline
\end{tabular}

Figure. 14. Main Part of Piston Object

\begin{tabular}{|c|c|c|}
\hline$\checkmark$ Main $\checkmark$ Options $\checkmark$ & & \\
\hline Attribute & Unit & Object Value \\
\hline Ignition Delay & & $3 \ldots$ \\
\hline Premixed Fraction & & $0.02 \ldots$ \\
\hline Tail Fraction & & $0.05 \ldots$ \\
\hline Premixed Duration & & $2 \ldots$ \\
\hline Main Duration & & $35 \ldots$ \\
\hline Tail Duration & & $40 \ldots$ \\
\hline Premixed Exponent & & $\operatorname{def}(=0.7) \ldots$ \\
\hline Main Exponent & & $\operatorname{def}(=0.9) \ldots$ \\
\hline Tail Exponent & & $\operatorname{def}(=1.5) \ldots$ \\
\hline
\end{tabular}

Figure. 15. Main Part of Combustion Object

\section{Injector System}

The diesel engine Direct Injection Compression Ignition (DICI), the type of data that must be inserted, includes the fuel temperature, fuel type, the angle of its appearance, and the fuel mass injected.

\begin{tabular}{|c|c|c|c|}
\hline$\checkmark$ Mass & \multirow{2}{*}{$\frac{\searrow \text { Timing } \searrow \text { Fluid } \searrow \text { Nozzle }}{\text { Attribute }}$} & \multicolumn{2}{|c|}{$\triangle$ Profile Settings $\triangle$ Profile $⿴ 囗$ Plots } \\
\hline & & Unit & Object Value \\
\hline \multicolumn{2}{|c|}{ Injected Mass } & See Cas... $\vee$ & [NJMASS] $\ldots$ \\
\hline \multirow{2}{*}{$\nabla$} & Air-to Fuel Ratio Limit Methodology & & TotalComposition \\
\hline & Air-to-Fuel Ratio Limit & & {$[A F R] \ldots$} \\
\hline \multicolumn{4}{|c|}{ Figure. 16. Mass Part of Injector } \\
\hline \multirow[t]{2}{*}{$\checkmark N$} & Mass $\leadsto$ Timing $\leadsto$ Fluid $\leadsto$ Nozzle & $\checkmark$ Profile Setting & gs $\leadsto$ Profile $⿴$ Plots \\
\hline & Attribute & Unit & Object Value \\
\hline \multicolumn{2}{|c|}{ Injection Timing } & $\operatorname{deg}$ & $-18 \ldots$ \\
\hline \multicolumn{4}{|c|}{ Source of Angle } \\
\hline $\mathrm{O}$ & \multicolumn{3}{|l|}{ Attached Cylinder } \\
\hline () & Part on Map & & $\operatorname{def} \ldots$ \\
\hline $\mathrm{O}$ & Driver Reference Object & & ign \\
\hline
\end{tabular}

Figure. 17. Timing Part of Injector

\begin{tabular}{|c|c|c|c|}
\hline$\checkmark$ Mass $\searrow$ Timing $\searrow$ Fluid & $\checkmark$ Nozzle & $\checkmark$ Profile Setting: & $\approx$ Profile $ه$ Plots \\
\hline Attribute & & Unit & Object Value \\
\hline Fluid Object & & & [BahanBakar] ... \\
\hline Injected Fluid Temperature & & K & $303.15 \ldots$ \\
\hline Vaporized Fluid Fraction & & & $0 \ldots$ \\
\hline
\end{tabular}

Figure. 18. Fluid Part of Injector

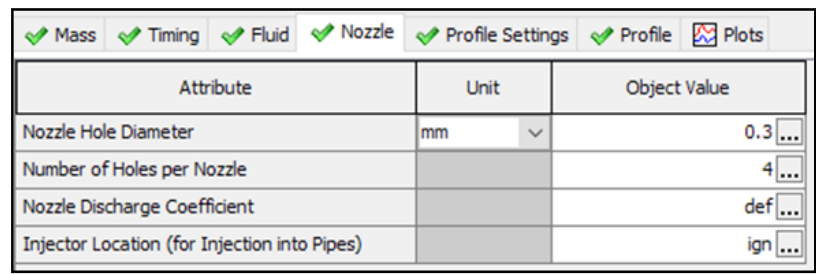

Figure. 19. Nozzle Part of Injector 


\begin{tabular}{|c|c|c|}
\hline Attri... & Time or Angle Array & Pressure or Mass Array \\
\hline 0 & & bar \\
\hline 1 & \begin{tabular}{|l|l|}
$-18.0 \ldots$ \\
\end{tabular} & $0.0 \ldots$ \\
\hline 2 & $-17.0 \ldots$ & $15.11111111 \ldots$ \\
\hline 3 & $-16.0 \ldots$ & $18.11111111 .$. \\
\hline 4 & $-15.0 \ldots$ & $22.11111111 \ldots$ \\
\hline 5 & $-14.0 \ldots$ & $27.11111111 \ldots$ \\
\hline 6 & $-13.0 \ldots$ & $33.11111111 .$. \\
\hline 7 & $-12.0 \ldots$ & $40.11111111 .$. \\
\hline 8 & $-11.0 \ldots$ & $48.11111111 .$. \\
\hline 9 & $-10.0 \ldots$ & $57.11111111 .$. \\
\hline 10 & $-9.0 \ldots$ & $67.11111111 .$. \\
\hline 11 & $-8.0 \ldots$ & $78.11111111 \ldots$ \\
\hline 12 & $-7.0 \ldots$ & $90.11111111 .$. \\
\hline 13 & $-6.0 \ldots$ & $103.1111111 .$. \\
\hline 14 & $-5.0 \ldots$ & $117.1111111 .$. \\
\hline 15 & $-4.0 \ldots \ldots$ & $132.1111111 .$. \\
\hline 16 & $-3.0 \ldots$ & $148.1111111 .$. \\
\hline 17 & $-2.0 \ldots$ & $165.1111111 .$. \\
\hline 18 & $-1.0 \ldots$ & $183.1111111 .$. \\
\hline 19 & $0.0 \ldots$ & $200.0 \ldots$ \\
\hline 20 & $1.0 \ldots$ & $183.1111111 \ldots$ \\
\hline 21 & $2.0 \ldots$ & $165.1111111 .$. \\
\hline 22 & $3.0 \ldots$ & $148.1111111 \ldots$ \\
\hline 23 & $4.0 \ldots$ & $132.1111111 \ldots$ \\
\hline 24 & $5.0 \ldots$ & $117.1111111 \ldots$ \\
\hline 25 & $6.0 \ldots$ & $103.1111111 \ldots$ \\
\hline 26 & $7.0 \ldots$ & $90.11111111 \ldots$ \\
\hline 27 & \begin{tabular}{ll|l|}
8.0 & $\ldots$ \\
\end{tabular} & $78.11111111 \ldots$ \\
\hline & $m a t$ & с \\
\hline
\end{tabular}

Figure. 20. Profile Part of Injector

3. Cranktrain

Diesel engine simulation Cranktrain explains the type of motor, cylinder arrangement, ignition sequence, the characteristics of the crankshaft, and other characteristics. Input data that need to be filled in the form of motor type, motor rotation, inertia, cylinder geometry, and ignition sequence.

\begin{tabular}{|l|l|r|}
\hline \multicolumn{1}{|c|}{ Attribute } & \multicolumn{1}{c|}{ Unit } & \multicolumn{2}{c|}{ Object Value } \\
\hline Engine Type & & 4-stroke \\
\hline Speed or Load Specification & & load \\
\hline Engine Speed & See Cas... & \\
\hline Engine Friction Object or FMEP & & \\
\hline Start of Cycle (CA at IVC) & & [RPM] \\
\hline
\end{tabular}

Figure. 21. Main Part of Cranktrain

\begin{tabular}{|c|c|c|}
\hline \multicolumn{3}{|l|}{ Main } \\
\hline Attribute & Unit & Object Value \\
\hline Constant part of FMEP & bar & $0.03 \ldots$ \\
\hline Peak Cylinder Pressure Factor & & $0.004 \ldots$ \\
\hline Mean Piston Speed Factor & $\operatorname{bar} /(\mathrm{m} / \mathrm{s}) \quad v$ & $0.053 \ldots$ \\
\hline Mean Piston Speed Squared Factor & $\operatorname{bar} /(\mathrm{m} / \mathrm{s} \ldots \vee$ & $0.0006 \ldots$ \\
\hline Engine Speed Upon Entering Friction Transition Band & RPM & $\operatorname{def}(=2.0) \ldots$ \\
\hline
\end{tabular}

Figure. 22. Main Part of Friction

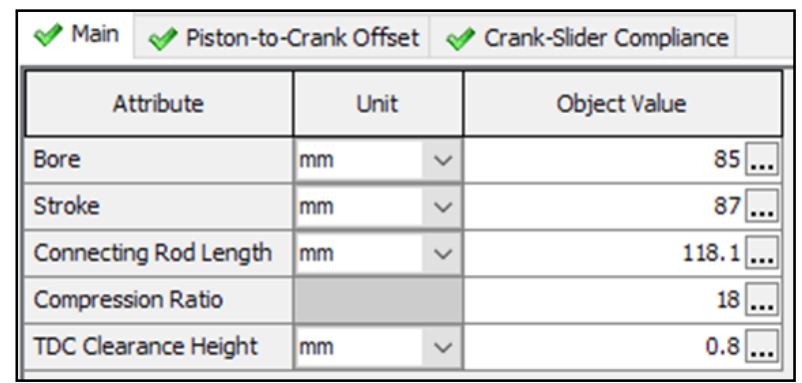

Figure. 23. Main Part of EngCylGeom

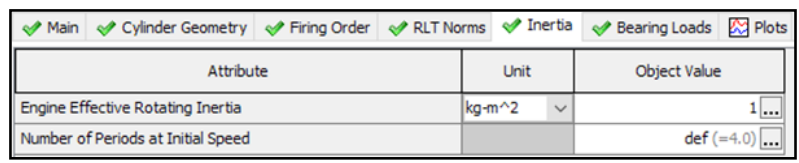

Figure. 24. Inertia Part of Cranktrain

4. Load Torque/ Dynamometer

The load Torque/dynamometer is a component that will be coupling with the crank train of the machine, and it serves to regulate the loading by changing the load value according to the desired research.

\begin{tabular}{|l|c|c|}
\hline \multicolumn{1}{|c|}{ Main } & \\
\hline Attribute & Unit & Object Value \\
\hline Torque & See Cas... $\vee$ & [BEBAN] $\ldots .$. \\
\hline
\end{tabular}

Figure. 25. Inertia Part of Cranktrain

C. In the inlet system of diesel engine simulation has several components that must be defined :

1. Inlet Environment

The inlet environment in the simulation of a diesel engine must make a condition of supporting boundary by determining the value of pressure, temperature, and air composition of the environment according to normal conditions.

\begin{tabular}{|c|c|c|c|c|}
\hline$\checkmark$ Main Options & Altitude and Humidity & 图 Plots & & \\
\hline & Attribute & & Unit & Object Value \\
\hline Pressure (Absolute) & & $b a$ & $\checkmark$ & $1 \ldots$ \\
\hline Temperature & & K & $\checkmark$ & $303.15 \ldots$ \\
\hline Composition & & & & air $\ldots$ \\
\hline
\end{tabular}

Figure. 26. Main Part of Inlet Environment

\begin{tabular}{||l|r|lr|}
\hline \multicolumn{1}{|l|}{$\checkmark$ Main $\checkmark$ Fluids } \\
\hline Attri... & Fluid Object & Mass or Volume Fraction \\
\hline 0 & & fraction & $\checkmark$ \\
\hline 1 & n2-vap & & 0.767 \\
\hline 2 & o-vap & \\
\hline
\end{tabular}

Figure. 27. Part of Composition Air in Simulation

2. Intake Runner System

Diesel engine simulation also has a connection in one circuit. The network link is a pipe that serves as a conduit between the Inlet Environment and the Intake Port. The Data required is basic geometry and material. 


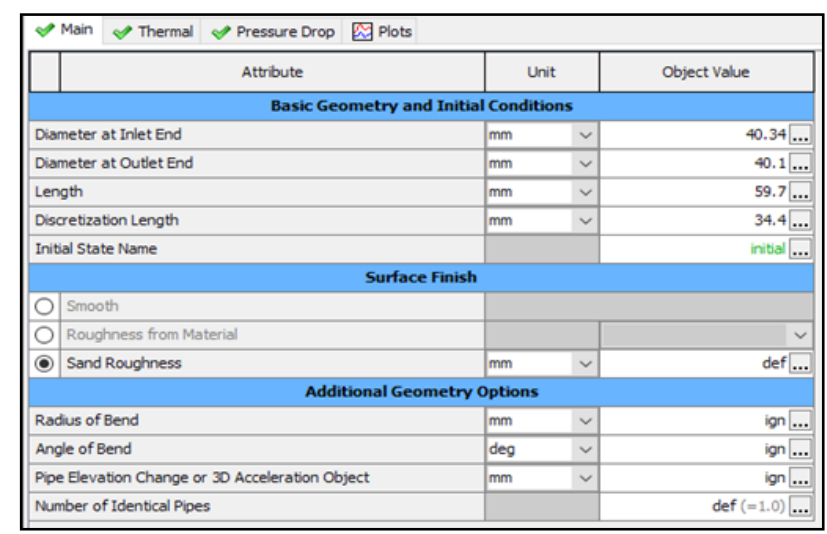

Figure. 28. Intrunner's Part of Basic Geometry and Initial Conditions

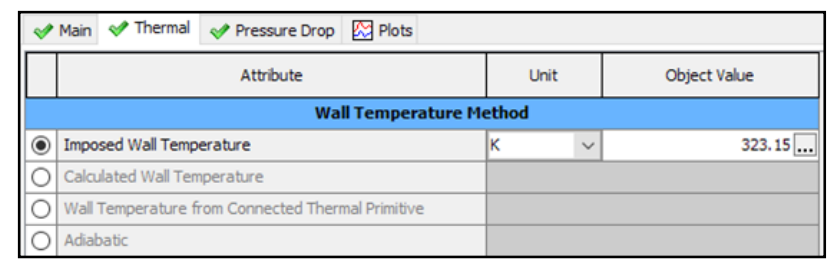

Figure. 29. Intrunner Part of Thermal

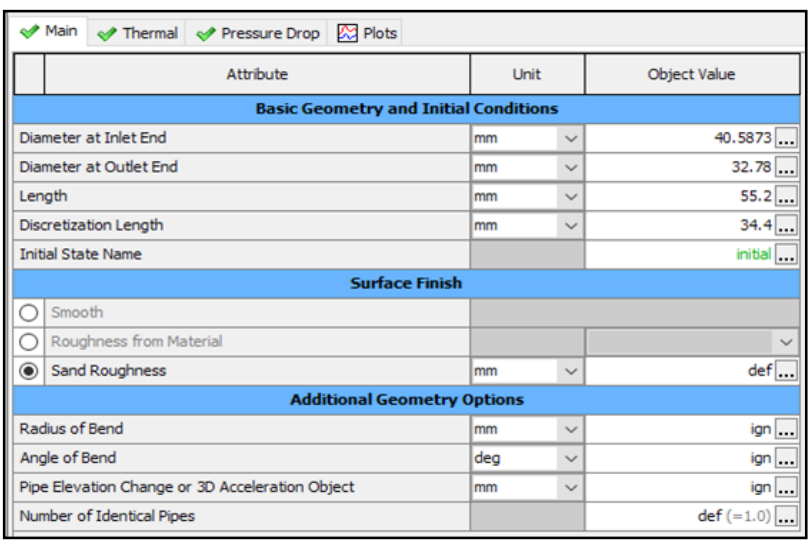

Figure. 30. Basic Geometry and Initial Conditions Part of

3. Intake Valve

$$
\text { Intport }
$$

The diesel engine intake valve simulation has a model of valves made by inserting valve diameter value, lash valve, timing cam angle, and lift array. This component serves to make the camshaft and valve characteristics of the diesel motor.

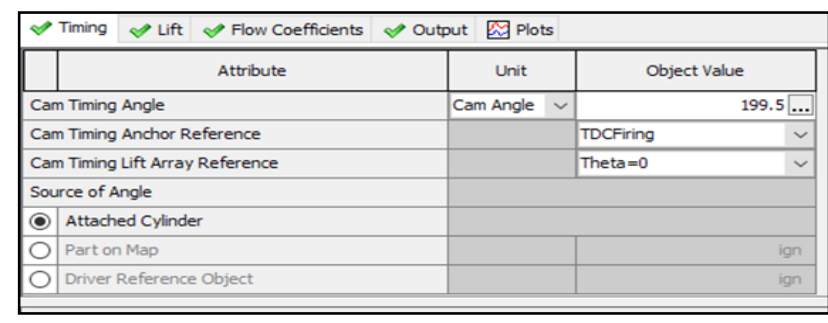

Figure. 31. Timing Part of Intake Valve

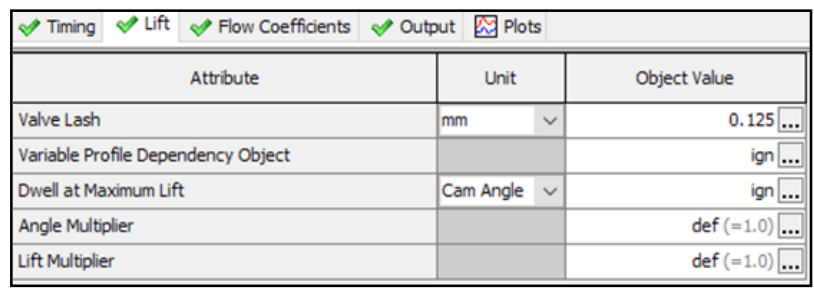

Figure. 32. Lift Part of Intake Valve

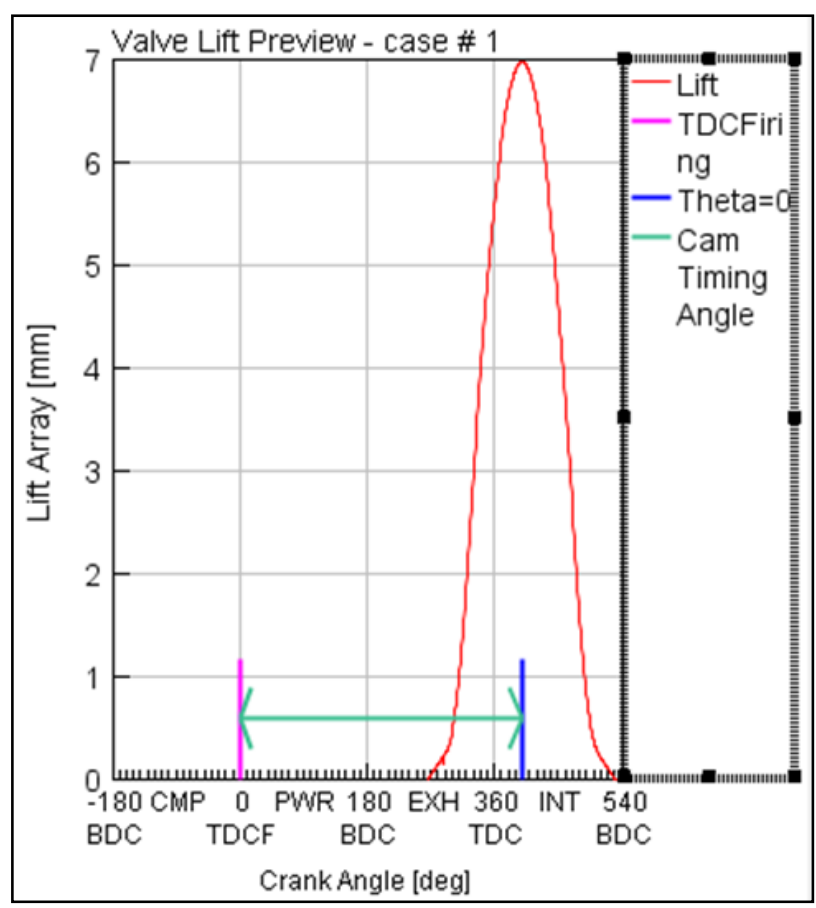

Figure. 33. Lift Array Part of Intake Valve

\section{4) Model Series of Diesel Engine Simulation Yanmar TF-85}

Object Data from diesel engine specification has been incorporated into the modeling of engine simulation. The simulation engine can already be run for simulating the performance of biodiesel fuel variations. Figure 35 shows the diesel engine simulation series.

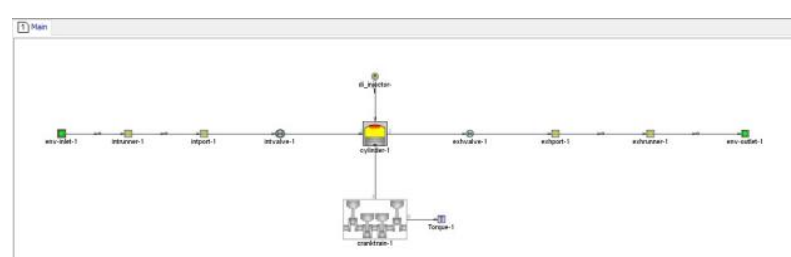

Figure. 34. Modeling diesel engine simulation Yanmar TF 85

\section{B. Simulated Fuel Variations}

This simulation uses the computing system of the setting in the simulation to create a variety of used cooking oil biodiesel B50-B90. The Data needed for the manufacture of fuel variations are the characteristics of the HSD and 
B100 obtained from the journal. In table 2 shows the fuel properties that will be simulated.

TABLE.2.

PROPERTIES VARIATIONS OF BIODIESEL AND HSD FUELS

\begin{tabular}{lllll}
\hline $\begin{array}{l}\text { Fuel } \\
\text { properties }\end{array}$ & $\begin{array}{l}\text { Density, } \\
\mathbf{k g} / \mathbf{m}^{3}\end{array}$ & $\begin{array}{l}\text { Lower } \\
\text { heating } \\
\text { value, } \\
\text { MJ/kg }\end{array}$ & $\begin{array}{l}\text { Critical } \\
\text { temperature, } \\
\text { K }\end{array}$ & $\begin{array}{l}\text { Critical } \\
\text { pressure, } \\
\text { bar }\end{array}$ \\
\hline HSD & 836 & 43,25 & 594,4 & 24,6 \\
B100 & 890,7 & 37,11 & 785,9 & 12,07 \\
B50 & 864,3 & 39,76 & 677,7 & 18,34 \\
B60 & 868.82 & 39,14 & 699,3 & 17,082 \\
B70 & 874,29 & 38,52 & 720,95 & 15,829 \\
B80 & 879,8 & 37,92 & 742,6 & 14,58 \\
B90 & 885,23 & 37,33 & 764,25 & 13,323 \\
\hline
\end{tabular}

\section{Validation engine simulation Diesel TF 85}

Calibration is used to validate diesel engine simulation Yanmar TF 85. Data needed to validate the Power, SFOC, and torque. Supporting data from calibration is obtained from simulated results compared to Data in accordance with the specifications of Yanmar diesel engine 85 . In table 4 is the result of a simulation that will be compared to engine specifications.

TABLE. 3. HSD SIMULATION CALIBRATION RESULTS

\begin{tabular}{cccl}
\multicolumn{4}{c}{ HSD SIMULATION CALIBRATION RESULTS } \\
\hline RPM & $\begin{array}{c}\text { POWER, } \\
\mathrm{kW}\end{array}$ & $\begin{array}{c}\text { TORQUE, } \\
\text { Nm }\end{array}$ & $\begin{array}{l}\text { BFSC, } \\
\text { gr/kW- } \\
\text { h }\end{array}$ \\
\hline 500 & 1,548 & 30.260 & 282.641 \\
600 & 1,978 & 31.478 & 268.282 \\
700 & 2,368 & 32.304 & 258.61 \\
800 & 2,752 & 32.847 & 250.717 \\
900 & 3,126 & 33.170 & 245.124 \\
1000 & 3.491 & 33.334 & 240.765 \\
1100 & 3.848 & 33.359 & 237.438 \\
1200 & 4.182 & 33.276 & 234.835 \\
1300 & 4.504 & 33.087 & 232.892 \\
1400 & 4.813 & 32.829 & 231.346 \\
1500 & 5.104 & 32.491 & 230.273 \\
1600 & 5.337 & 32.092 & 229.523 \\
1700 & 5.631 & 31.632 & 229.114 \\
1800 & 5.868 & 31.129 & 228.916 \\
1900 & 6.084 & 30.580 & 228.946 \\
2000 & 6.238 & 29.997 & 229.151 \\
2100 & 6.457 & 29.360 & 229.662 \\
2200 & 6.613 & 28.706 & 230.254 \\
\hline & & &
\end{tabular}

TABLE. 4

COMPARISON OF RESULTS CALIBRATION AND ENGINE SPECIFICATIONS

\begin{tabular}{|c|c|c|c|c|}
\hline \multirow{3}{*}{$\begin{array}{l}1600 \\
\text { RPM }\end{array}$} & \multirow[b]{2}{*}{ Calibration } & $\begin{array}{l}\text { Power, } \\
\mathrm{kW}\end{array}$ & $\begin{array}{l}\text { Torque, } \\
\mathrm{Nm}\end{array}$ & $\begin{array}{l}\text { SFOC, } \\
\text { g/kW- } \\
\mathrm{h}\end{array}$ \\
\hline & & - & 32,09 & - \\
\hline & $\begin{array}{l}\text { Annual } \\
\text { book }\end{array}$ & - & 33,73 & - \\
\hline \multirow{2}{*}{$\begin{array}{l}2200 \\
\text { RPM }\end{array}$} & Calibration & 6,61 & - & 230,25 \\
\hline & $\begin{array}{l}\text { Annual } \\
\text { book }\end{array}$ & 6,33 & - & 229,31 \\
\hline Error & & $4,4 \%$ & $4,8 \%$ & $4,07 \%$ \\
\hline
\end{tabular}

\section{Simulation of Engine Diesel}

In the modeling of this diesel engine, use the GTPower simulation to know the performance value of the Yanmar diesel engine 85 by using used cooking oil biodiesel fuel at full load conditions.

\section{RESULT AND DISCUSSION}

In this research, a performance test will be conducted to determine the effect of biodiesel B50-B100 on the simulation of the Yanmar TF85-MH diesel engine. This research uses GT-POWER software. The results of this experiment will later determine the performance in using B50-B100 fuel and will be compared with biofuels that have been traded in the market, namely HSD. The rotation used in this experiment starts at 600 RPM, 800 RPM, 1000 RPM, 1200 RPM, 1400 RPM, 1600 RPM, 1800 RPM, 1900 RPM, 2000 RPM, 2100 RPM, and 2200 RPM under $100 \%$ full load condition.

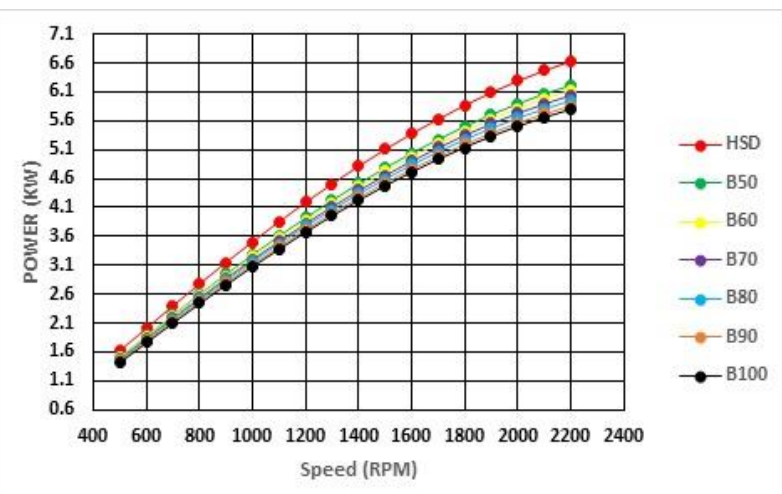

Figure 35. Graphic Power Vs. RPM at Full Load Conditions

Based on Figure 1 is the comparison graph between Engine round to power in full load conditions for all types of fuel. This chart is a comparison of engine round performance to power on all types of fuel. This value is derived from the lowest SFOC point described in previous graphs. Obtained the highest or maximum obtained from a high rotation of 2200 RPM on each fuel, at a speed of 2200 RPM, HSD has the highest power 
value of $6.63 \mathrm{~kW}$, in the same round, the fuel B50 has the second-highest power value of $6.21 \mathrm{Kw}$, followed by B60 fuel has the third-highest power of $6.12 \mathrm{Kw}$, on B70 fuel has a power of $6.04 \mathrm{Kw}$, on the B80 fuel has a power of $5.95 \mathrm{~kW}$, the $\mathrm{B} 90$ fuel has a power of $5.87 \mathrm{~kW}$, and on the material, B100 has a power of $5.78 \mathrm{KW}$. Furthermore, at a round of 1800 RPM seen in the image 1, HSD fuel still has the highest power value of $5.87 \mathrm{~kW}$, in the same round conditions, fuel B50 has the secondhighest power value of $5.50 \mathrm{Kw}$, followed by the B60 fuel to have the third-highest power of $5.42 \mathrm{Kw}$, on the B70 fuel has a power of $5.35 \mathrm{Kw}$, on B80 fuel has a power of $5.28 \mathrm{Kw}$, on the B90 fuel has a power of 5.20 $\mathrm{kW}$, and in B100 material has a power of $5.13 \mathrm{~kW}$. In a round of 1000 RPM seen in the image 1, HSD fuel still has the highest power value of $3.50 \mathrm{~kW}$; in the same round conditions, fuel $\mathrm{B} 50$ has the second-highest power value of $3.28 \mathrm{Kw}$, followed by the $\mathrm{B} 60$ fuel has the thirdhighest power of $3.24 \mathrm{Kw}$, on the fuel B70 has a power of $3.20 \mathrm{Kw}$, on $\mathrm{B} 80$ fuel has a power of $3.16 \mathrm{Kw}$, on the $\mathrm{B} 90$ fuel has a power of $3.11 \mathrm{~kW}$, and in B100 material has a power of $3.07 \mathrm{~kW}$.

In low rounds of 600 RPM seen in the image graph 1, HSD fuel still has the highest power value of $2.00 \mathrm{~kW}$, in the same round conditions, fuel B50 has the secondhighest power value of $1.88 \mathrm{Kw}$, followed by the B60 fuel has the third-highest power of $1.86 \mathrm{Kw}$, on B70 fuel has a power of $1.83 \mathrm{Kw}$, on B80 fuel has a power of 1.81 $\mathrm{Kw}$, on the B90 fuel has a power of $1.79 \mathrm{~kW}$, and in B100 material has a power of $1.76 \mathrm{~kW}$. From the observation of the graph as a whole, it can be concluded that the larger the engine rotation then, the greater the power is generated, and the power generated HSD is greater than the power generated from the biodiesel mixing fuel in each round. According to research [8], the heating value of a biodiesel fuel lower than the HSD fuel results in a decrease in the resulting power output. The larger the value of the biodiesel mixture then the lower the calorie value, which causes the resulting power to fall down.

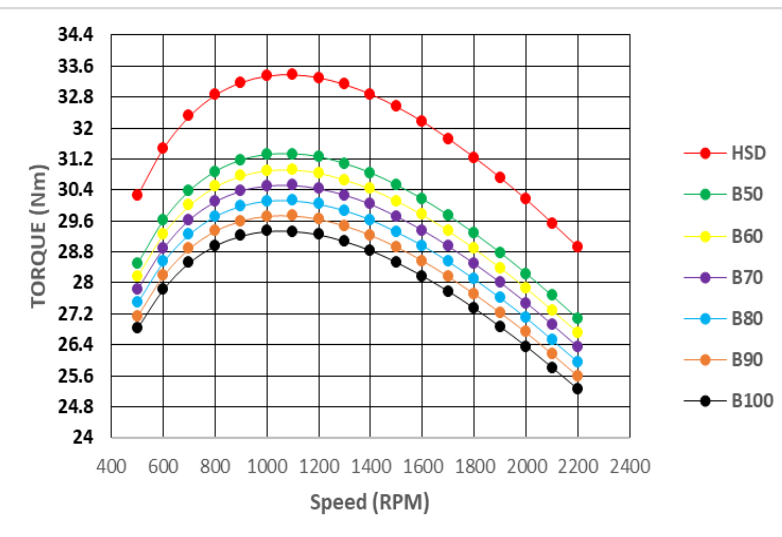

Figure 36. Graphic Torque Vs. RPM at Full Load Conditions

Based on figure 2, which is the comparison graph between Engine round to torque in full load conditions for all types of fuel, the graph is a comparison of the maximum torque value with a rotation on each type of fuel, where the largest torque value is obtained at a load of $100 \%$. At a low round of 600 RPM, it was seen that the HSD fuel had the highest torque value of $31.47 \mathrm{Nm}$, in the same round conditions as the B50 fuel had the second-highest torque value of $29.61 \mathrm{Nm}$, followed by the B60 fuel having the third-highest torque of 29.26 $\mathrm{Nm}$, on the B70 fuel having a torque of $28.89 \mathrm{Nm}$, on a B80 fuel having a torque of $28.54 \mathrm{Nm}$, on the B90 fuel has a torque of $28.18 \mathrm{Nm}$, and on the B100 material has a torque of $27.8 \mathrm{Nm}$. In the $1000 \mathrm{RPM}$ round, it was seen that the HSD fuel had the highest torque value of 33.34 $\mathrm{Nm}$, in the same rotation condition that B50 fuel had the second-highest torque value of $31.31 \mathrm{Nm}$, followed by the B60 fuel having the third-highest torque of 30.90 $\mathrm{Nm}$, on the fuel B70 had a torque of $30.50 \mathrm{Nm}$, on B80 fuel had a torque of $30.11 \mathrm{Nm}$, on the B90 fuel has a torque of $29.72 \mathrm{Nm}$, and on the B100 material has a torque of $29.33 \mathrm{Nm}$. In the $1100 \mathrm{RPM}$ round, it was seen that the HSD fuel had the highest torque value of 33.38 $\mathrm{Nm}$, in the same rotation condition that B50 fuel had the second-highest torque value of $31.33 \mathrm{Nm}$, followed by the B60 fuel having the third-highest torque of 30.92 $\mathrm{Nm}$, on the fuel B70 had a torque of $30.52 \mathrm{Nm}$, on B80 fuel had a torque of $30.12 \mathrm{Nm}$, on the B90 fuel has a torque of $29.72 \mathrm{Nm}$, and on the B100 material has a torque of $29.33 \mathrm{Nm}$. In the $1800 \mathrm{RPM}$ round, it was seen that the HSD fuel had the highest torque value of 31.24 $\mathrm{Nm}$, in the same rotation condition that B50 fuel had the second-highest torque value of $29.27 \mathrm{Nm}$, followed by the B60 fuel having the third-highest torque of 28.88 $\mathrm{Nm}$, on the fuel B70 had a torque of $28.49 \mathrm{Nm}$, on B80 fuel had a torque of $28.10 \mathrm{Nm}$, on the B90 fuel has a torque of $27.71 \mathrm{Nm}$, and on the B100 material has a torque of $27.33 \mathrm{Nm}$. Furthermore, there is a maximum round of 2200 RPM seen that the HSD fuel has the highest torque value of $28.91 \mathrm{Nm}$, in the same round conditions as the B50 fuel has the second-highest torque value of $27.07 \mathrm{Nm}$, followed by the B60 fuel has the third-highest torque of $26.33 \mathrm{Nm}$, on the fuel B70 has a torque of $26.33 \mathrm{Nm}$, on $\mathrm{B} 80$ fuel has a torque of 25.96 $\mathrm{Nm}$, on the B90 fuel has a torque of $25.60 \mathrm{Nm}$, and on the B100 material has a torque of $25.23 \mathrm{Nm}$.

This is in accordance with the calculations of the software and theories where torque is derived from the calculation between power comparisons and RPM, resulting in a maximum increase at $1100 \mathrm{RPM}$. Where on the chart also seen an increase from low RPM to 1100, and dropped from 1100 to 2200 . These torque tendencies will continue to decline as the RPM increases after the peak point and RPM reduction before the peak point. It is according to the formula that has been formulated in the software. From the overall graph observation, the results gained that HSD fuel is a greater torque value than $\mathrm{B} 50, \mathrm{~B} 60, \mathrm{~B} 70, \mathrm{~B} 80, \mathrm{~B} 90, \mathrm{~B} 100$ in each round. According to research [8]. The increase in biodiesel joints is the decrease in the torque value that is generated by the heat value contained in each fuel. The 
value of the Calor is lower; then, the torque result will be decreased.

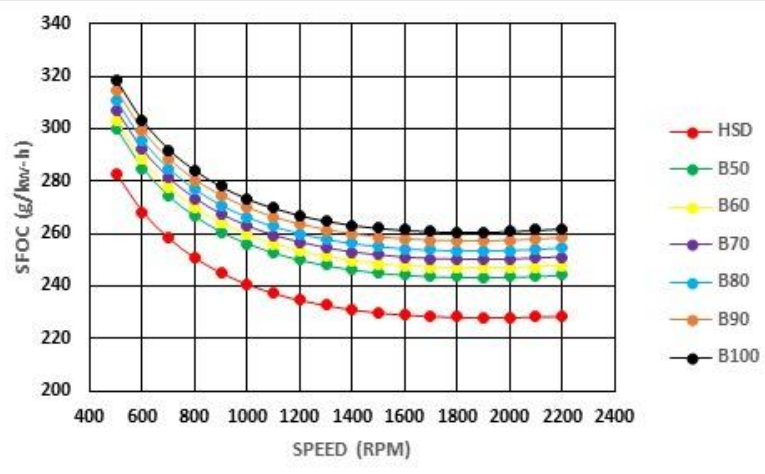

Figure 37. Graphic SFOC Vs. RPM at Full Load Conditions

Based on Figure 3, the comparison graph between Engine round to SFOC on Full Load condition. On the graphs described earlier, that the lowest SFOC value is seen at a maximum load of $100 \%$ Full Load. At a low round of $600 \mathrm{RPM}$, it appears that HSD fuel has the lowest SFOC value of $268.26 \mathrm{~g} / \mathrm{kWh}$, in the same rotation conditions as B50 fuel has the second-lowest SFOC value of $284.91 \mathrm{~g} / \mathrm{kWh}$, followed by fuel B60 has the third-lowest SFOC of $288.29 \mathrm{G} / \mathrm{kWh}$, on B70 fuel has an SFOC of $291.94 \mathrm{~g} / \mathrm{kWh}$, on the fuel B80 has an SFOC of $295.40 \mathrm{~g} / \mathrm{kWh}$, on the fuel B90 has an SFOC of $299.14 \mathrm{~g} / \mathrm{kWh}$, and on B100 material has an SFOC of $302.98 \mathrm{~g} / \mathrm{kWh}$. In the $1000 \mathrm{RPM}$ round, it appears that the HSD fuel has the lowest SFOC value of 240.72 $\mathrm{g} / \mathrm{kWh}$, in the same rotation conditions as B50 fuel has the second-lowest SFOC value of $256.19 \mathrm{~g} / \mathrm{kWh}$, followed by fuel B60 has the third-lowest SFOC of 259.5 $\mathrm{G} / \mathrm{kWh}$, on B70 fuel has an SFOC of $262.88 \mathrm{~g} / \mathrm{kWh}$, on the fuel B80 has an SFOC of $266.23 \mathrm{~g} / \mathrm{kWh}$, on the fuel B90 has an SFOC of $269.73 \mathrm{~g} / \mathrm{kWh}$, and on B100 material has an SFOC of $273.25 \mathrm{~g} / \mathrm{kWh}$. In the 1800 RPM round, it appears that the HSD fuel has the lowest SFOC value of $228.25 \mathrm{~g} / \mathrm{kWh}$, in the same rotation conditions as B50 fuel has the second-lowest SFOC value of $243.47 \mathrm{~g} / \mathrm{kWh}$, followed by fuel $\mathrm{B} 60$ has the third-lowest SFOC of $246.78 \mathrm{G} / \mathrm{kWh}$, on B70 fuel has an SFOC of $250.11 \mathrm{~g} / \mathrm{kWh}$, on the fuel B80 has an SFOC of $253.57 \mathrm{~g} / \mathrm{kWh}$, on the fuel $\mathrm{B} 90$ has an SFOC of 257.08 $\mathrm{g} / \mathrm{kWh}$, and on B100 material has an SFOC of 260.62 $\mathrm{g} / \mathrm{kWh}$.

At a high round of 2200 RPM, it appears that HSD fuel has the lowest SFOC value of $228.57 \mathrm{~g} / \mathrm{kWh}$. In the same rotation conditions, B50 fuel has the second-lowest SFOC value of $244.26 \mathrm{~g} / \mathrm{kWh}$, followed by fuel B60 has the third-lowest SFOC of $247.62 \mathrm{G} / \mathrm{kWh}$, on B70 fuel has an SFOC of $251.06 \mathrm{~g} / \mathrm{kWh}$, on the fuel B80 has an SFOC of $254.62 \mathrm{~g} / \mathrm{kWh}$, on the fuel B90 has an SFOC of $258.22 \mathrm{~g} / \mathrm{kWh}$, and on B100 material has an SFOC of $261.91 \mathrm{~g} / \mathrm{kWh}$. From the observation of the chart as a whole, it can be concluded that the fuel HSD, as well as variations of the used cooking oil biodiesel fuel the larger round of the Engine then the lower the SFOC value, but at 2000 RPM increased the value of SFOC on fuel HSD, B50, B60, B70, B80, B90 because the Engine is experiencing overload. It is different from the B100 fuel, first experiencing an overloaded engine at 1900 RPM. The results obtained that the fuel HSD lower the value of SFOC than B50, B60, B70, B80, B90, B100 in each round. According to research [14]. The Calor value contained on the HSD is higher than the biodiesel calor value. The more the replenishment of the biodiesel value then the smaller the Calor value resulting in biodiesel fuel need more fuel to get the same energy, resulting in a higher SFOC biodiesel value than the HSD.

\section{CONCLUSION}

Based on the results of the simulated performance test on HSD fuel, B50, B60, B70, B80, B90, B100 waste cooking oil biodiesel, it can be withdrawn as follows:

1) The SFOC value gained in performance tests can be concluded that the lowest SFOC value is located in full load at each round, and the higher the engine rotation, the lower the SFOC value. In full load conditions with maximum RPM the lowest SFOC value is HSD fuel with a value of $228.57 \mathrm{gr} / \mathrm{kw}$, further comparison of the value of SFOC HSD with B50 up about $6.8 \%$, B60 up $8.3 \%$, B70 up $9.83 \%$, B80 up $11.4 \%$, B90 up $12.9 \%$, and B100 up $14.5 \%$.

2) The resulting power is influenced by engine rotation. The higher the engine rotation, the greater the power generated by the Engine. When the condition is a full load at maximum RPM, the largest power is generated on the type of HSD fuel, followed by a variety of used cooking oil biodiesel fuel. HSD's generated power is $6.63 \mathrm{~kW}$, further comparison of HSD power values with B50 down $6.38 \%$, B60 dropped 7.6\%, B70 dropped 8.9\%, B80 dropped $10.2 \%$, B90 11.4\%, and B100 dropped by $12.7 \%$.

3) The resulting torque increased at $500 \mathrm{RPM}$ to a maximum torque point at 1100 RPM after it decreased torque value to maximum RPM. The results obtained that $\mathrm{HSD}$ fuel is greater torque value than B50, B60, B70, B80, B90, B100 in each round. Torque value HSD at maximum RPM full load condition i.e., $28.9 \mathrm{Nm}$, further comparison with the B50 down $6.3 \%$, B60 dropped 7.6\%, B70 dropped $8.9 \%$, B80 dropped $10.2 \%$, B90 dropped $11.4 \%$, and B100 dropped $12.7 \%$.

\section{REFERENCES}

[1] Misra, R., \& Murthy, M. (2011). Blending of additives with biodiesels to improve the cold flow properties, combustion and emission performance in a compression ignition engine a review. Renew Sustain Energy, 15:2413-22.

[2] Sheehan, J., Duffield, J., Garboski, M., \& Shapouri, H. (1998). An overview of biodiesel and petroleum diesel life cycles. A report by US Department of Agriculture and Energy, 1-35.

[3] Panji, R. (2019, Desember Senin). Rilis Bahan Bakar B30, Presiden Jokowi Perintah Kembangkan Hingga B100. Retrieved from 
Carmudi.co.id: https://www.carmudi.co.id/journal/rilis-bahan-bakarb30-presiden-jokowi-perintah-kembangkan-hingga-b100

[4] Suwarsono, W. (2008). Sintesis Biodiesel dari Minyak Biji Ketapang Yang Tumbuh di Kapus UI Depok. Valensi, vol.1,no.2, 4452.

[5] Raharjo, S. (2007). Analisa Performa Mesin Disel dengan Bahan Bakar Biodiesel dari Minyak Jarak Pagar. Prosiding Seminar Nasional Teknologi, B1-B6.

[6] Yuniarsi, K. (2007). Coco Metyl Ester (Cocodiesel) Sebagai Bahan Bakar Pengganti Diesel fuel. Jurnal Akta

[7] Aziz, I. (2012). Uji Performance Mesin Diesel Menggunakan Biodiesel Dari Minyak Goreng Bekas. 2-3.

[8] Sugozu, I., Eryilmaz, T., Ors, I., \& Solmaz, O. (2011). Biodiesel production from animal fat-palm oil blend and performance analysis of its effects on a single cylinder diesel engine. Energy Education Science and Technology Part A: Energy Science and Research.

[9] Haryono, Fairus, S., Sari, Y., \& Rakhmawati, I. (Januari, 2010). Pengolahan Minyak Goreng Kelapa Sawit Bekas menjadi Biodiesel Studi Kasus: Minyak Goreng Bekas dari KFC Dago Bandung. Pengembangan Teknologi Kimia untuk Pengolahan Sumber Daya Alam Indonesia.

[10] McCrady, J., Hansen, A., \& Fon Lee, C. (2007). Modeling Biodiesel Combustion Using GT-Power. ASABE.

[11] Semin, RA, B., \& AR, I. (2007). Effect of Engine Performance For Four Stroke Diesel Engine Using SImulation. Proceeding The 5th International Conferences .

[12] Semin, RA, B., \& AR, I. (2008). Investigation of diesel engine performance based on simulation. American Journal of Applied Sciences, (6) 610-617.

[13] Gamma Technologies (2004). GT-POWER User's Manual 6.1, Gamma Technologies Inc.

[14] Nirmala, N., Dawn, S., \& Harindra, C. (2019). Analysis of performance and emission characteristics of Waste cooking oil and Chlorella variabilis MK039712.1 biodiesel blends in a single cylinder, four strokes diesel engine. www.elsevier.com/locate/renene.

[15] Magfirotunnisa, Gunawan, \& Saksono, P. (2018). ANALISIS PERBANDINGAN PENGGUNAAN BAHAN BAKAR DIESEL FUEL DENGAN BIODIESEL B15 DAN B20 TERHADAP PERFORMANSI. SNITT- Politeknik Negeri Balikpapan.

[16] Technologies Gamma Engine Perfomance Tutorial [Book],Westmont : [s.n.],2016

[17] www.wartaekonomi.com/indicator, 2006.

[18] Yudisaputra, A. (2017). Analisis Pengaruh Angka Iodin Terhadap Proses Pembakaran Pada Motor Diesel.

[19] Muwaffaq, A. H. (2016). Prediksi Performa Marine Diesel 4 Langkah 93 KW dengan Metode Simulasi.

[20] Kimindo, vol 3, no.1, 17-20 